\title{
DISTRIBUCIÓN OPTIMIZADA DE VOLÚMENES LIMITADOS DE AGUA DE RIEGO Y SU EFECTO SOBRE EL RENDIMIENTO Y LA CALIDAD DE LA CEBADA CERVECERA
}

\author{
Pardo, J.J. ${ }^{1}(\mathrm{P})$, Léllis, B.C. ${ }^{2}$, Martínez-Romero, A. $^{3}$, Tarjuelo, J.M. ${ }^{4},{\text { Domínguez, } A{ }^{5}}^{5}$
}

\author{
${ }^{1}$ Ingeniero Agrónomo, Investigador; Centro Regional de Estudios del Agua (CREA), UCLM, \\ Ctra. de Las Peñas km. 3.2, 02071 Albacete; josejesus.pardo@uclm.es \\ ${ }^{2}$ Ingeniero Agrónomo, Investigador; Centro Regional de Estudios del Agua (CREA), UCLM, \\ Ctra. de Las Peñas km. 3.2, 02071 Albacete; brunolellismaria@yahoo.com.br \\ ${ }^{3}$ Dr. Ingeniero Agrónomo, Profesor Asociado; EIIAB, Departamento de Mecánica Aplicada e \\ Ingeniera de Proyectos, UCLM; angel.mromero@uclm.es \\ ${ }^{3}$ Dr. Ingeniero Agrónomo, Catedrático de Universidad; Departamento de Producción Vegetal \\ y Tecnología Agraria, UCLM; jose.tarjuelo@uclm.es \\ ${ }^{5}$ Dr. Ingeniero Agrónomo, Profesor Contratado Doctor; Departamento de Producción Vegetal \\ y Tecnología Agraria, UCLM; alfonso.dominguez@uclm.es
}

\section{Resumen}

En Castilla-La Mancha (C-LM), la cebada es un cultivo con bajas necesidades de agua de riego (lámina neta, $I n=2.500 \mathrm{~m}^{3} \mathrm{ha}^{-1}$ ) y con una gran diferencia de rendimiento entre secano y regadío. Sin embargo, por la escasez de recursos hídricos, las dotaciones limitadas de agua, y el alto coste de la energía, los agricultores se están planteando aplicar riego deficitario a este cultivo. El objetivo de este trabajo es determinar, para las condiciones de C-LM, las estrategias de riego deficitario controlado que, con volúmenes limitados de agua de riego, maximizan el rendimiento de la cebada cervecera sin que la calidad del producto se vea afectada. Se han desarrollado ensayos de campo considerado 5 volúmenes de agua disponible (llimitada, $1 \mathrm{ln}, 0.9 \mathrm{ln}, 0.8 \mathrm{ln}$, y $0.7 \mathrm{ln}$ ), optimizando los calendarios de riegos combinando las metodologías "año meteorológico típico" (Tipical Meteorological Year,TMY) y "riego deficitario optimizado por etapas" (Optimized Regulated Deficit Irrigation, ORDI) con el modelo MOPECO. Se ha conseguido una buena distribución del agua disponible a lo largo de la campaña, sin alcanzar un estrés severo global en ninguna de las etapas de desarrollo, logrando un alto rendimiento sin decremento de la calidad, pese a desconocer las condiciones climáticas en las que se iba a desarrollar el cultivo. Los rendimientos obtenidos fueron similares a los estimados al inicio de la campaña y los parámetros de calidad medidos, excelentes en todos los tratamientos.

\section{1- Introducción, objetivos}

Castilla-La Mancha (C-LM) es el segundo productor de cebada en España con una producción de 2.784.281 Tm (28\% nacional). En dicha comunidad se cultivan 857.267 ha, de las que 97.383 ha son en regadío (MAGRAMA, 2016). Pese a ser una región con escasez de recursos hídricos, la gran diferencia de rendimiento entre secano y regadío (hasta 3 veces mayor) junto con las bajas necesidades de agua riego de este cultivo (unos $2.500 \mathrm{~m}^{3} \mathrm{ha}^{-1}$ ), justifican la implantación de este cultivo en regadío. Sin embargo, el incremento de los costes de producción, especialmente de la energía eléctrica necesaria para el bombeo del agua subterránea y el funcionamiento de los sistemas de riego presurizados (Moreno et al., 2010), así como el tener dotaciones limitadas de agua por 
campaña de riego, está ocasionando que los agricultores se planteen la posibilidad de aplicar riego deficitario a este cultivo.

Si el grano de la cebada alcanza unos determinados índices de calidad, como ciertos calibres y especialmente determinado contenido en proteína, éste puede ser empleado en la fabricación de malta para cerveza. La empresa Intermalta España, situada en Albacete, que se dedica al malteado de cebada para la elaboración de cerveza, fija el contenido de proteína del grano en torno al 9-12.5\% siendo el óptimo 11.5-12\%.

El objetivo principal de este trabajo es determinar, para las condiciones de C-LM, las estrategias de riego deficitario controlado que, con volúmenes limitados de agua de riego, maximizan el rendimiento de la cebada cervecera sin que la calidad del producto se vea afectada. Para ello se han combinado las metodologías del "año meteorológico típico" (Tipical Meteorological Year, TMY) (Domínguez et al., 2013), que determina las características típicas ambientales de una zona, y del "riego deficitario optimizado por etapas" (Optimized Regulated Deficit Irrigation, ORDI) (Domínguez et al., 2012), que determina mediante optimizaciones sucesivas a lo largo de la campaña la distribución de una cantidad limitada de agua en cada una de las fases de desarrollo del cultivo en función de las condiciones ambientales previstas por el TMY y la disponibilidad de agua de riego. Ambas metodologías se han implementado en el modelo MOPECO y se han validado con ensayos de campo durante la campaña 2015.

\section{2- Materiales y métodos}

Durante la campaña 2015 se realizaron ensayos de campo en los que se analizó el efecto de 5 tratamientos de riego sobre el rendimiento y los parámetros de calidad de la cosecha. Los tratamientos planteados fueron: uno sin déficit, y los otros cuatro con distintos volúmenes máximos de agua de riego disponible, correspondientes al 100 (T100), 90 (T90), 80 (T80), y 70\% (T70) de las necesidades netas de riego en cebada para las condiciones climáticas de un año meteorológico típico (TMY) intermedio (fijadas en $2500 \mathrm{~m}^{3} / \mathrm{ha}=\mathrm{T} 100$ ). Este valor se obtuvo tras la calibración de los datos requeridos por el modelo MOPECO para la cebada en las condiciones de C-LM, aplicando la metodología TMY como se explicará más adelante.

Los ensayos tuvieron lugar en la finca experimental del Centro Integrado de Formación Profesional situado en Aguas Nuevas (Albacete), que consistieron en cuatro repeticiones de cada tratamiento en parcelas de $2.5 \times 18 \mathrm{~m}$. distribuidas al azar (Figura 1), salvo en los tratamientos sin déficit y T100, que tuvieron 3.

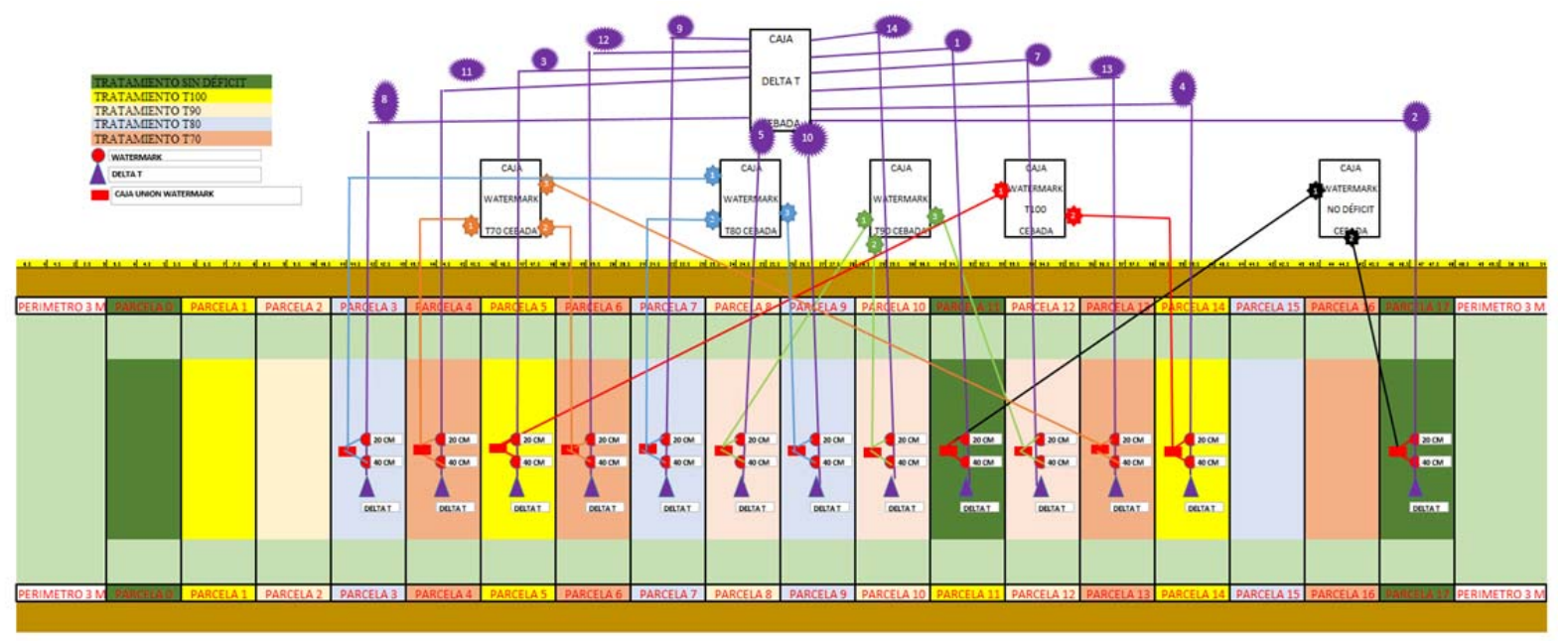

Figura 1. Esquema del ensayo

El agua se aplicó mediante un sistema de riego localizado por goteo de marco cuadrado (0.5 x $0.5 \mathrm{~m}$ de separación entre ramales y emisores) dotado de emisores autocompensantes 
con un caudal nominal de $3.8 \mathrm{~L} / \mathrm{h}$. En cada sector de riego, que corresponde con un tratamiento, se instaló un caudalímetro de pulsos de precisión para controlar el volumen de agua aplicada en cada riego. Además se realizaron evaluaciones de la uniformidad de aplicación de agua del sistema de riego antes de la siembra y periódicamente a lo largo del ciclo de cultivo.

\section{1- Calibración del modelo MOPECO. Establecimiento de las necesidades de riego netas.}

MOPECO utiliza la ecuación propuesta por Stewart et al. (1977) para estimar el rendimiento del cultivo que relaciona la evapotranspiración real y la máxima $\left(E T_{a} / E T_{m}\right)$ en las diferentes etapas de crecimiento. Doorenbos y Kassam (1979) consideran cuatro etapas de crecimiento de acuerdo a la distinta sensibilidad de los cultivos a déficit hídrico (desarrollo vegetativo, floración, formación de rendimiento y período de maduración). El factor de respuesta del rendimiento del cultivo, $\mathrm{Ky}$, expresa lo sensible que es el cultivo al déficit hídrico en cada etapa de crecimiento. Cuando $\mathrm{ET}_{\mathrm{a}}<\mathrm{ET}_{\mathrm{m}}$, la planta sufre estrés por déficit de agua, lo que puede causar una pérdida en el rendimiento [rendimiento real $\left(\mathrm{Y}_{\mathrm{a}}\right)<$ máximo rendimiento $\left.\left(Y_{m}\right)\right]$.

$$
\mathrm{Y}_{\mathrm{a}}=\mathrm{Y}_{\mathrm{m}} \prod_{\mathrm{k}=1}^{4}\left(1-\mathrm{K}_{\mathrm{y}_{\mathrm{k}}}\left(1-\frac{\mathrm{ET}_{\mathrm{a}_{\mathrm{k}}}}{\mathrm{ET}_{\mathrm{m}_{\mathrm{k}}}}\right)\right)
$$

donde $Y_{a}$ e $Y_{m}$, son el rendimiento real y máximo del cultivo $\left(\mathrm{kg} \mathrm{ha}^{-1}\right) ; \mathrm{k}$, es etapa de crecimiento real; $\mathrm{K}_{\mathrm{yk}}$, es el factor de respuesta del rendimiento por etapa de crecimiento [desarrollo vegetativo (i), que incluye el establecimiento (Domínguez et al., 2012); (ii) la floración, (iii) formación de rendimiento; y (iv) periodo de maduración]; $\mathrm{ET}_{\mathrm{a}} \mathrm{y} \mathrm{ET}_{\mathrm{m}}$, indican las evapotranspiraciones acumuladas real y máxima en cada etapa de crecimiento $\mathrm{Ky}(\mathrm{mm})$.

$\mathrm{La} \mathrm{ET}_{\mathrm{m}}$ diaria se calcula multiplicando el $\mathrm{Kc}$ diario por la evapotranspiración de referencia $\left(\mathrm{ET}_{\mathrm{o}}\right.$ ) diaria (Allen et al., 1998). $\mathrm{ET}_{\mathrm{a}}$ diaria requiere un balance diario del contenido de agua en el suelo para su estimación, el balance de agua en el suelo diario se calcula como la diferencia entre las entradas (precipitaciones y riego) y salidas ( $E T_{a}$ y percolación profunda) (Domínguez et al., 2011).

Para la simulación de la cebada también se necesita la duración de las etapas de Kc y Ky en grados día acumulados (GDD). MOPECO utiliza el método de doble triangulación, que requiere dos parámetros para el cálculo GDD (Sevacherian et al., 1977): TL (temperatura umbral mínima para el desarrollo) y TU (temperatura umbral máxima a la que la tasa de desarrollo comienza a disminuir). Para establecer los valores de TL y TU en el área de estudio, se han analizado y comparado con los valores propuestos por otros autores en áreas similares (Canadá; Etiopia, Italia, Siria, EE.UU.). TL puede variar de 0 a $10^{\circ} \mathrm{C}$, y TU de 20 a $38^{\circ} \mathrm{C}$ (Kirby et al., 1982; López-Bellido, 1991; Juskiw et al., 2001; Araya et al., 2010; Abrha et al., 2012). Se seleccionó la combinación de valores con la menor desviación estándar (SD) y menor coeficiente de variación (CV) en función de los datos de campo.

Para determinar la duración de cada etapa Kc y Ky en términos de GDD, se utilizaron los resultados de los ensayos de campo realizados durante las campañas 2002, 2004, 2011, 2012 y 2013 en la finca experimental de Las Tiesas, perteneciente al Instituto Técnico Agronómico Provincial de Albacete (ITAP) complementados con 28 seguimientos fenológicos de cultivo de cebada llevados a cabo por el Servicio Integral de Asesoramiento al Regante (SIAR), perteneciente al CREA, durante los años 2002-2010 por toda C-LM. La escala fenológica utilizada en ambos casos fue Biologische Bundesanstalt, Bundessortenamt y la Industria Química (BBCH) (Bleiholder et al., 2001).

Una vez calibrado MOPECO y con la ayuda de la metodología TMY, se determinaron las necesidades de agua de riego típicas del cultivo (lámina neta, In). En base a estas necesidades, se plantearon los cuatro tratamientos de riego con volumen limitado de agua citados anteriormente $(100,90,80$, y $70 \%$ de In). A partir de los datos climáticos ambientales registrados por la estación agroclimática completa ubicada en el campo de ensayos, de los registros de los sensores de humedad en el suelo, y de la combinación de MOPECO con las 
metodologías ORDI y TMY, se estableció el nivel de déficit objetivo ( $\left.E T_{a} / E T_{m}\right)$ para cada etapa de crecimiento. Así, se determinó el calendario de riegos para cada uno de los tratamientos propuestos, sin que se superase en ningún caso el volumen de agua disponible prefijado.

\section{2- Seguimiento del consumo de agua por parte del cultivo}

En tres de cada cuatro repeticiones se instalaron sensores de humedad en el suelo del tipo Watermark ${ }^{\circledR}$ (tensiómetro) y PR2 ${ }^{\circledR}$ (humedad volumétrica) para determinar la evolución de la humedad del suelo a distintas profundidades (en los tratamientos sin déficit y T100, en dos de cada tres repeticiones).

Cada parcela dispuso de 2 Watermark ${ }^{\circledR}$ a 20 y $40 \mathrm{~cm}$ de profundidad y una sonda PR2 ${ }^{\circledR}$ con sensores a 10, 20, 30 y $40 \mathrm{~cm}$ de profundidad.

\section{3- Seguimiento del desarrollo del cultivo.}

Durante el desarrollo fenológico del cultivo, se realizaron análisis fisiológicos (conductancia estomática y actividad fotosintética), se tomaron muestras periódicas para establecer el índice de área foliar y la evolución de la biomasa, así como fotografías RGB y térmicas. También se realizaron análisis de suelo para registrar la evolución de sus características físico-químicas.

Al acabar la campaña, se determinó el rendimiento final, se calculó la eficiencia en el uso de agua de riego, y se analizaron parámetros de calidad en colaboración con la empresa Intermalta España (ubicada en Albacete y perteneciente a la multinacional Malteurop, dedicada a la obtención de malta para la elaboración de cerveza) tales como: calibres, porcentaje de humedad, contenido en proteína del grano y capacidad de germinación.

\section{3- Resultados y discusión}

Se considera que un modelo está calibrado cuando la diferencia entre los rendimientos observados y simulados es igual o menor a un $10 \%$, y cuando el porcentaje de rendimientos simulados que cumplan el requisito anterior es igual o superior al 70\% (Farahani et al., 2009; Heng et al., 2009).

Para determinar los GDD acumulados de cada etapa de desarrollo, los valores de TL y TU que alcanzaron menor variabilidad en términos estadísticos fueron: 2 y $28^{\circ} \mathrm{C}$. Estos valores son idénticos a los propuestos por Abrha et al. (2012) y muy similares a los propuestos por otros autores como López-Bellido (1991), Juskiw et al. (2001), Qureshi et al. (2009), y Araya et al. (2010). En la Tabla 1 se recogen los valores de GDD acumulados en cada etapa de Kc y Ky para la cebada bajo las condiciones de C-LM.

La Tabla 2 muestra los valores de Kc y Ky así como el rendimiento potencial utilizados en MOPECO.

Tabla 1. GDD acumulados para las etapas de Kc y Ky.

\begin{tabular}{|c|c|c|c|c|c|c|c|c|}
\hline & \multicolumn{4}{|c|}{ Etapas de Kc } & \multicolumn{4}{|c|}{ Etapas de Ky } \\
\hline & Etapa 1 & Etapa 2 & Etapa 3 & Etapa 4 & Etapa 1 & Etapa 2 & Etapa 3 & Etapa 4 \\
\hline Media & 290.28 & 744.48 & 1087.18 & 1449.51 & 645.28 & 981.22 & 1186.09 & 1449.51 \\
\hline $\mathrm{SD}^{(1)}(\%)$ & 46.43 & 57.16 & 64.37 & 69.38 & 73.29 & 64.43 & 58.90 & 69.38 \\
\hline $\mathrm{CV}^{(2)}(\%)$ & 15.99 & 7.68 & 5.92 & 4.79 & 11.36 & 6.57 & 4.97 & 4.79 \\
\hline
\end{tabular}

(1) SD: Desviación estándar; ${ }^{(2)} \mathrm{CV}$ : Coeficiente de variación

Tabla 2. Valores de $\mathrm{Kc}, \mathrm{Ky}$, e $\mathrm{Y}_{\mathrm{m}}$ para la cebada bajo condiciones de C-LM.

Valores calibrados y validados para la cebada 


\begin{tabular}{|c|c|c|c|}
\hline Etapa & $\mathbf{K c}$ & $\mathbf{K y}$ & \multirow{2}{*}{$\mathbf{Y}_{\mathbf{m}}$ (kg/ha) } \\
\hline Etapa 1 & 0.3 & 0.2 & \\
\cline { 1 - 2 } Etapa 2 & $0.3-1.15$ & 0.55 & \multirow{2}{*}{9000} \\
\hline Etapa 3 & 1.15 & 0.30 & \\
\hline Etapa 4 & $1.15-0.45$ & 0.2 & \\
\hline
\end{tabular}

La Figura 2 muestra la evolución de los valores de Kc y Ky para el año 2015 en Albacete.

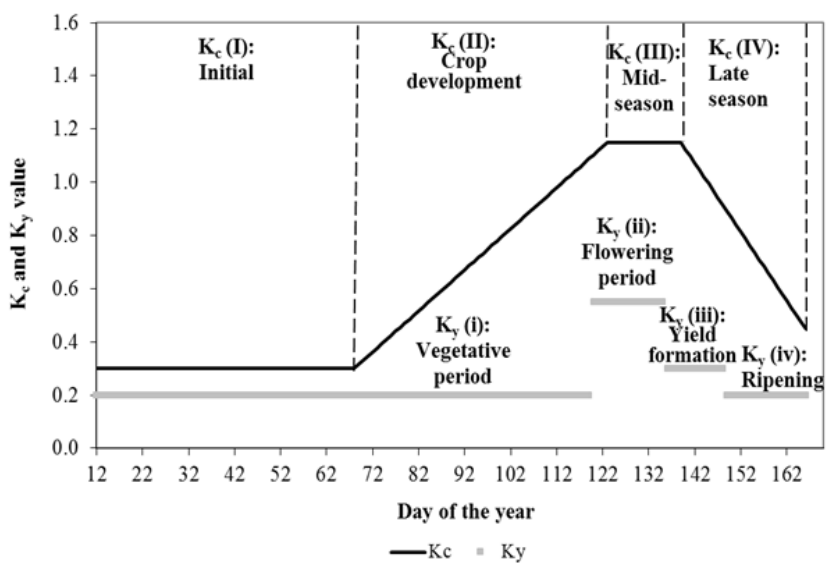

Figura 2. Evolución de los valores de Kc y Ky por etapas (año 2015).

En la Figura 3, en la gráfica superior, se puede apreciar la evolución del agua en el suelo simulada por MOPECO, y en la parte de abajo, los registros de los Watermark ${ }^{\circledR}$ para una parcela del T80. Se observa claramente que el modelo ha simulado muy bien las condiciones de agua en el suelo, ya que, aunque no se están representando las mismas unidades, las tendencias son similares. Por otra parte también se advierte que los objetivos de déficit planteados al principio de cada etapa no han coincidido con los conseguidos en algunas etapas, esto es debido principalmente a la distribución de las precipitaciones del año 2015, y en menor medida al resto de condiciones agroclimáticas (Tabla 4).

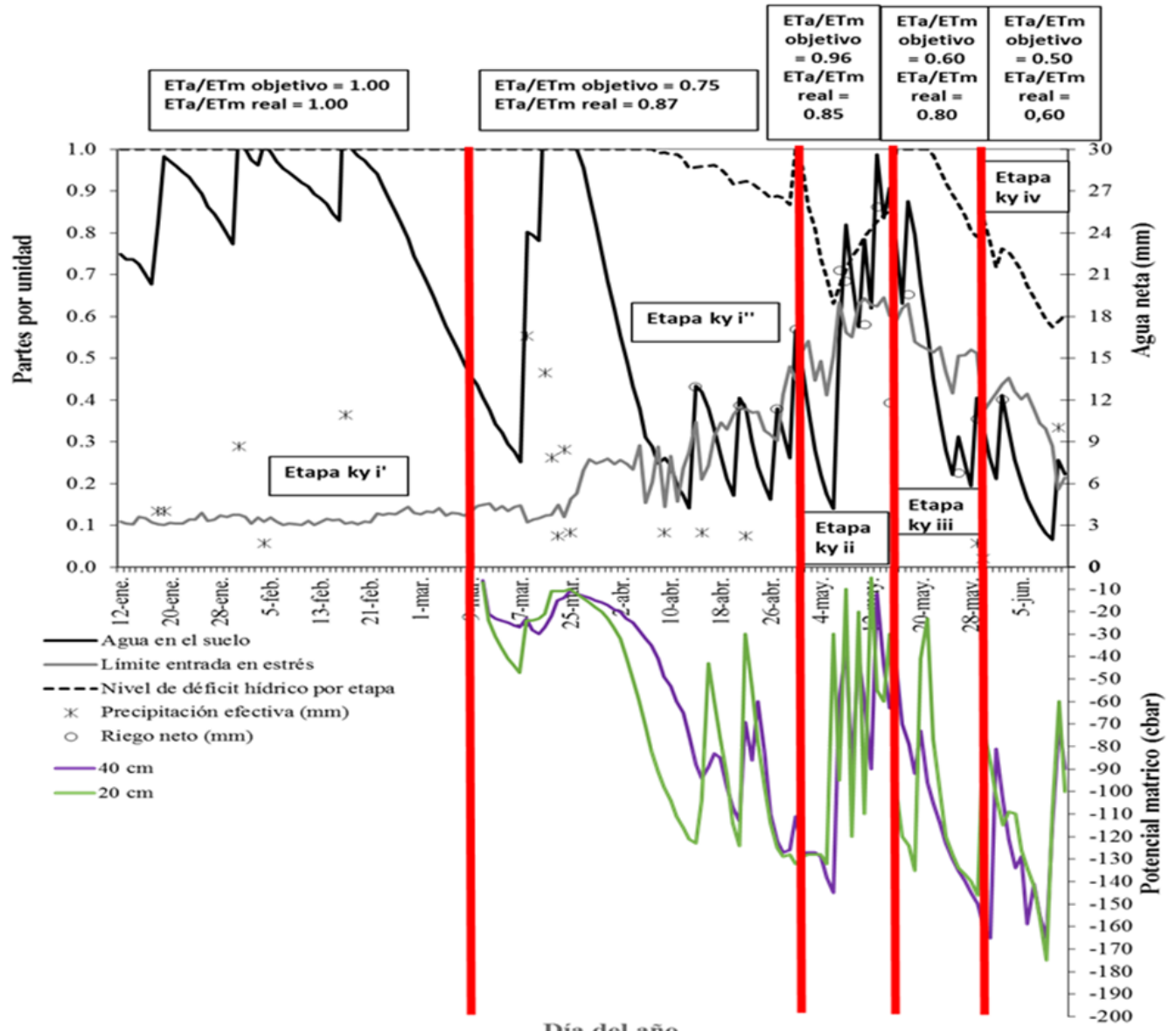


Figura 3. Evolución del agua en el suelo simulada por MOPECO para el tratamiento T80. Abajo, la evolución del agua en el suelo a 20 y $40 \mathrm{~cm}$ registrada por los Watermark ${ }^{\circledR}$.

Tabla 4. Riegos,ETa/ETm objetivos y reales por etapas Ky y tratamientos

\begin{tabular}{|c|c|c|c|c|c|c|c|c|c|c|c|c|c|c|c|}
\hline \multirow[b]{2}{*}{ Etapa } & \multicolumn{3}{|c|}{$\begin{array}{l}\text { Tratamiento Sin } \\
\text { déficit }\end{array}$} & \multicolumn{3}{|c|}{ Tratamiento T100 } & \multicolumn{3}{|c|}{ Tratamiento T90 } & \multicolumn{3}{|c|}{ Tratamiento T80 } & \multicolumn{3}{|c|}{ Tratamiento T70 } \\
\hline & $\begin{array}{l}\mathrm{ET}_{\mathrm{a}} / \\
\mathrm{ET}_{\mathrm{m}} \\
\text { obj. }\end{array}$ & $\begin{array}{l}\mathrm{ET}_{\mathrm{a}} \mathrm{l} \\
\mathrm{ET}_{\mathrm{m}} \\
\text { real }\end{array}$ & $\begin{array}{c}\text { Riego } \\
\text { neto } \\
(\mathrm{mm})\end{array}$ & $\begin{array}{l}\mathrm{Cl}_{\mathrm{a}} \mathrm{a}_{\mathrm{m}} \\
\mathrm{ET}_{\mathrm{m}} \\
\text { obj. }\end{array}$ & $\begin{array}{l}\mathrm{ET}_{\mathrm{a}} / \\
\mathrm{ET}_{\mathrm{m}} \\
\text { real }\end{array}$ & $\begin{array}{l}\text { neto } \\
(\mathrm{mm})\end{array}$ & $\begin{array}{l}\mathrm{ET}_{\mathrm{a}} \mathrm{l} \\
\mathrm{ET}_{\mathrm{m}} \\
\text { obj. }\end{array}$ & $\begin{array}{l}\mathrm{ET}_{\mathrm{a}} / \\
\mathrm{ET}_{\mathrm{m}} \\
\text { real }\end{array}$ & $\begin{array}{c}\text { Riego } \\
\text { neto } \\
(\mathrm{mm})\end{array}$ & $\begin{array}{l}\mathrm{ET}_{\mathrm{a}} / \\
\mathrm{ET}_{\mathrm{m}} \\
\text { obj. }\end{array}$ & $\begin{array}{l}\mathrm{ET}_{\mathrm{a}} / \\
\mathrm{ET}_{\mathrm{m}} \\
\text { real }\end{array}$ & $\begin{array}{l}\text { Riego } \\
\text { neto } \\
(\mathrm{mm})\end{array}$ & $\begin{array}{l}\mathrm{ET}_{\mathrm{a}} / \\
\mathrm{ET}_{\mathrm{m}} \\
\text { obj. }\end{array}$ & $\begin{array}{l}\mathrm{ET}_{\mathrm{a}} l \\
\mathrm{ET}_{\mathrm{m}} \\
\text { real }\end{array}$ & $\begin{array}{c}\text { Riego } \\
\text { neto } \\
(\mathrm{mm})\end{array}$ \\
\hline $1^{\prime}$ & 1.00 & 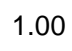 & مח0 & 1.00 & O & 0.00 & 100 & , & 0.00 & 100 & 0 & 0.00 & 1.00 & 1.00 & 0.00 \\
\hline $1 "$ & חר & 100 & 66.30 & 1.00 & 00 & 66.30 & 0.7 & $087-1-1-1$ & 35.90 & 0.75 & 0.87 & 3620 & 0.75 & 0.86 & 3.20 \\
\hline 2 & 00 & 0.99 & 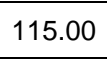 & 1.00 & 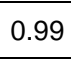 & 0 & 0.9 & 0.9 & 119.50 & $0 . c$ & 0.85 & 14.60 & 0.8 & .84 & 00 \\
\hline 3 & 1.00 & 0.99 & 69.30 & 1.00 & 0.99 & 69.30 & 0.76 & 0.81 & 26.10 & 0.60 & 0.81 & 26.50 & 0.54 & 0.65 & 23.90 \\
\hline 4 & 00 & 00 & 0 & 00 & 0.67 & 0.00 & 0.77 & 0.87 & 60 & 0.50 & 0.60 & 22 & 0.50 & 0.44 & 13.20 \\
\hline $\begin{array}{c}\text { Global/ } \\
\text { Total }\end{array}$ & 00 & .00 & 285.60 & .00 & 0.94 & 0.60 & 0.86 & 0.87 & 225.10 & 0.79 & 0.82 & 200.20 & 0.73 & 0.76 & 175.30 \\
\hline
\end{tabular}

En la primera etapa de Ky no hubo diferencias significativas en los calendarios de riego de los tratamientos T90, T80 y T70. Las $\mathrm{ET}_{\mathrm{a}} / \mathrm{ET}_{\mathrm{m}}$ objetivo hasta entonces fueron similares para estos tres tratamientos (el T100 siempre busca una $\mathrm{ET}_{\mathrm{a}} / \mathrm{ET}_{\mathrm{m}}$ objetivo de 1, es decir, no estrés). Es a partir de la etapa 2 de Ky cuando empezaron a distinguirse diferencias en las $\mathrm{ET}_{\mathrm{a}} / \mathrm{ET}_{\mathrm{m}}$ objetivo, acentuándose en las dos siguientes. No aparecieron diferencias en la evolución de la biomasa y del LAI hasta que el cultivo se encontraba en la etapa 2 de Ky (primera quincena de mayo) tal y como se aprecia en la Figura 4.
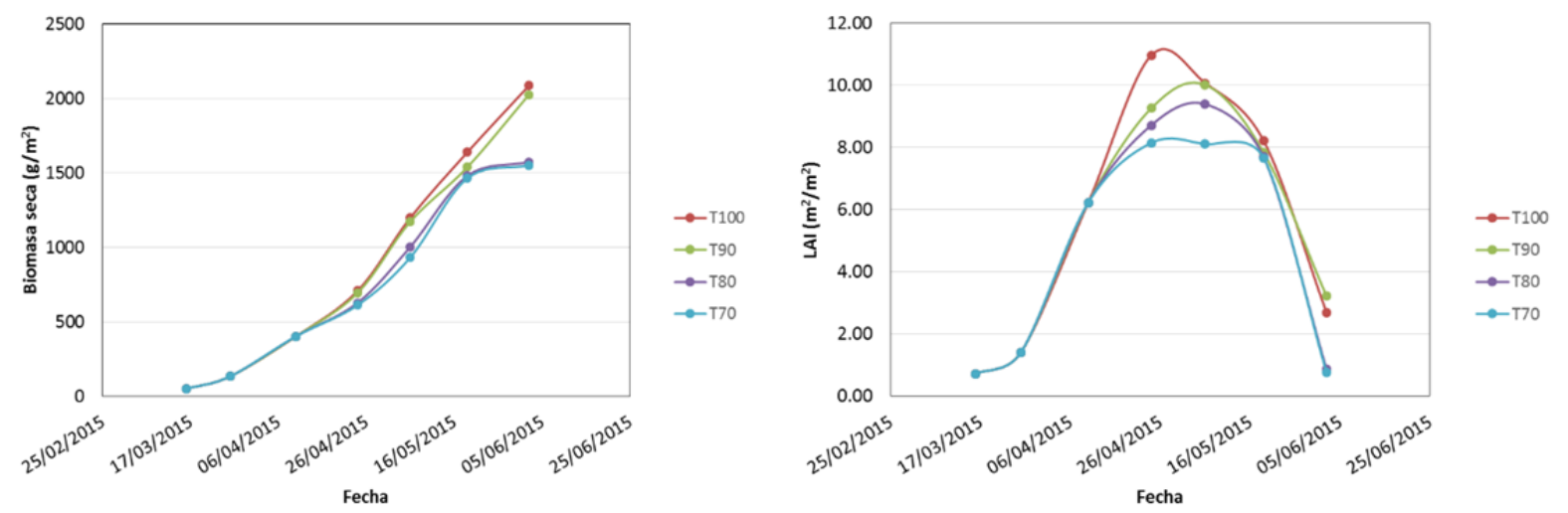

Figura 4. Evolución de la biomasa seca y del Índice de Área Foliar (LAI) en los tratamientos con volumen limitado de agua.

En cuanto a la resistencia estomática, sí que aparecieron diferencias entre tratamientos. En los tratamientos con mayores déficits la resistencia fue mayor, tal y como se aprecia en la Figura 5. El tratamiento T100 mantuvo una resistencia muy baja y prácticamente constante durante todo el periodo de medidas, el resto de tratamientos siguió la tendencia esperada. Cabe destacar que durante la etapa 2 de Ky, la más sensible al déficit, la resistencia ha sido similar en todos los tratamientos, eso es debido a que se buscaba la $\mathrm{ET}_{a} / \mathrm{ET}_{\mathrm{m}}$ mayor posible en todos ellos. Aunque al final de dicha etapa sí que aparecieron diferencias en la $E T_{a} / E T_{m}$ global, el que la resistencia haya sido igual se puede justificar porque en los días previos a 
la medida hubo muchos riegos, y la $\mathrm{ET}_{\mathrm{a}} / \mathrm{ET}_{\mathrm{m}}$ diaria para el momento de la medida era 1 en todos los casos.

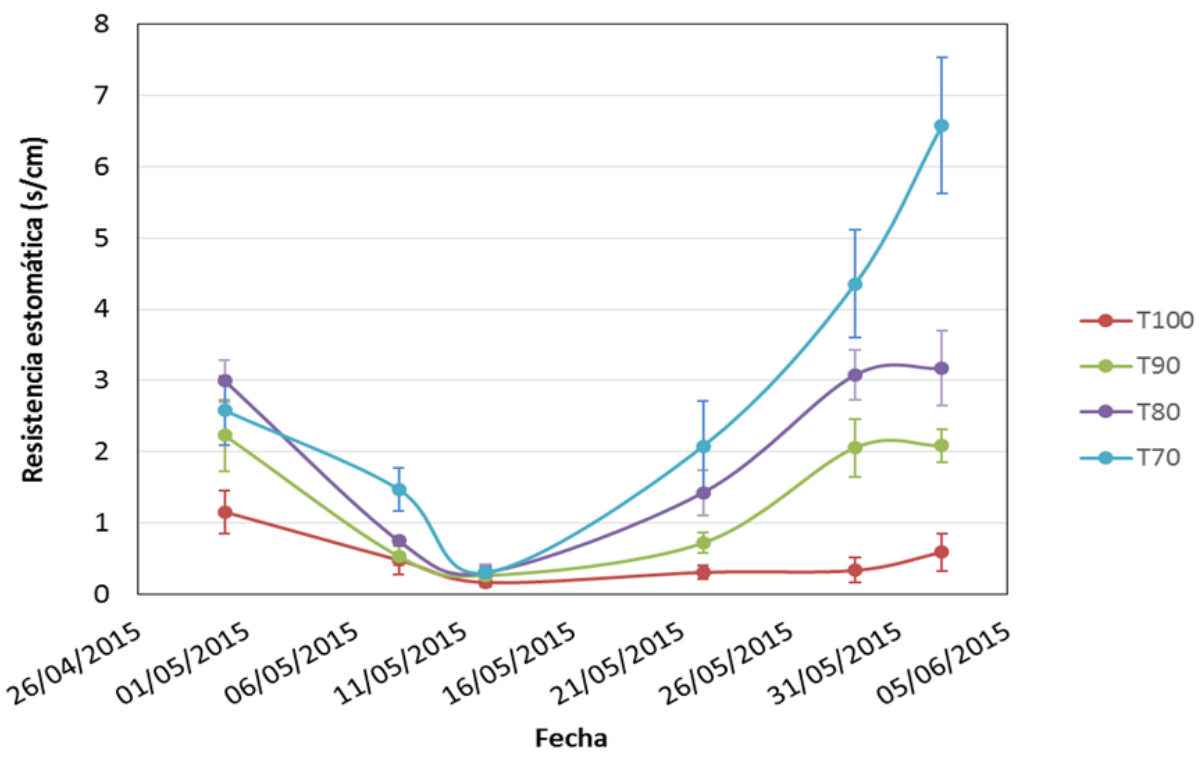

Figura 5. Evolución de la resistencia estomática $(\mathrm{s} / \mathrm{cm})$ de los cuatro tratamientos con volumen limitado de agua de riego.

La actividad fotosintética siempre fue mayor en los tratamientos con mayor volumen de agua disponible (Figura 6).

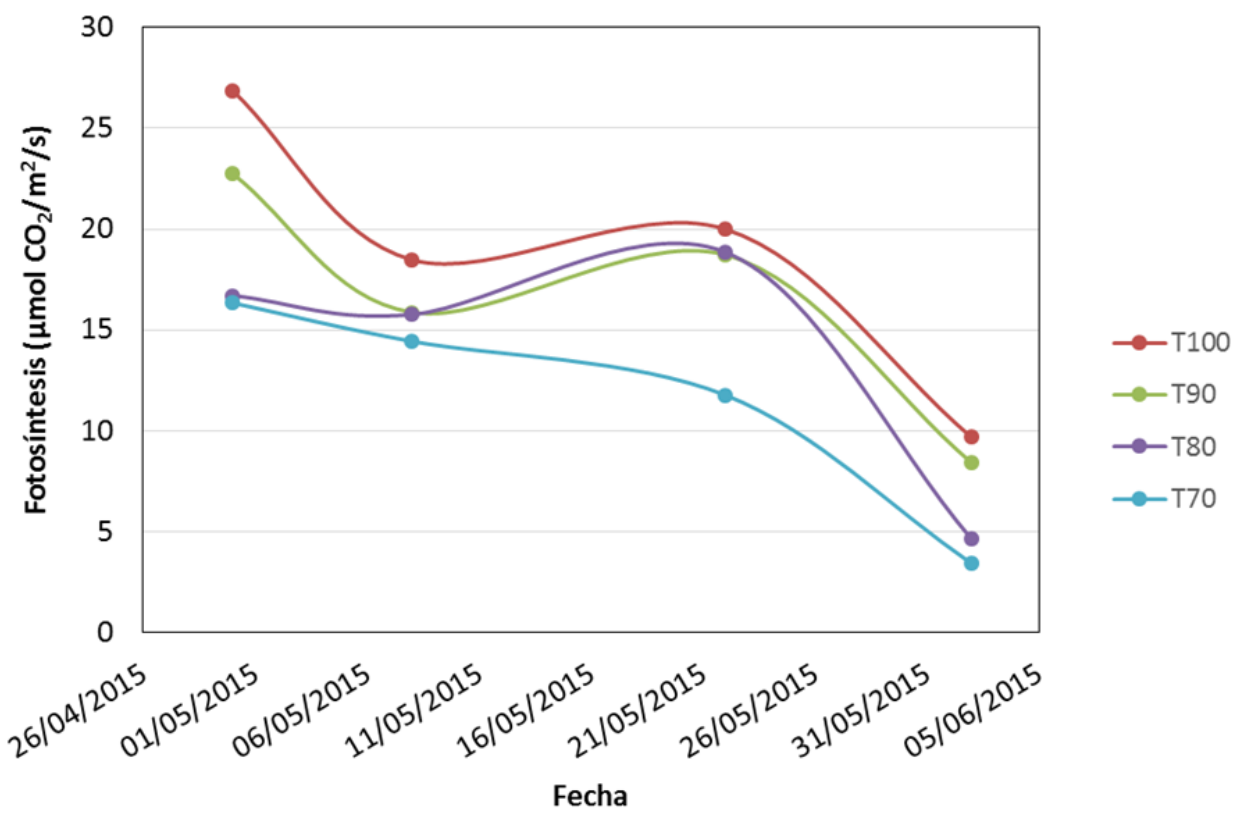

Figura 6. Evolución de la actividad fotosintética. 
Una vez que comienza la senescencia, la tasa fotosintética comienza a caer describiendo una curva. Según la Figura 6, esa curva no se aprecia en los tratamientos T100, T90 y T80. Esto se puede justificar porque la semana anterior a la medida del día 08/05, el sistema de bombeo se estropeó, las temperaturas fueron muy altas y las plantas estuvieron expuestas a un estrés mucho más severo del previsto. Por tanto, los registros de tasa fotosintética para ese día fueron más bajos de lo esperado.

En las fotos térmicas se observó que la temperatura de las hojas y espiga fue mayor en las parcelas con los tratamientos de menor agua de riego disponible (Figura 7).
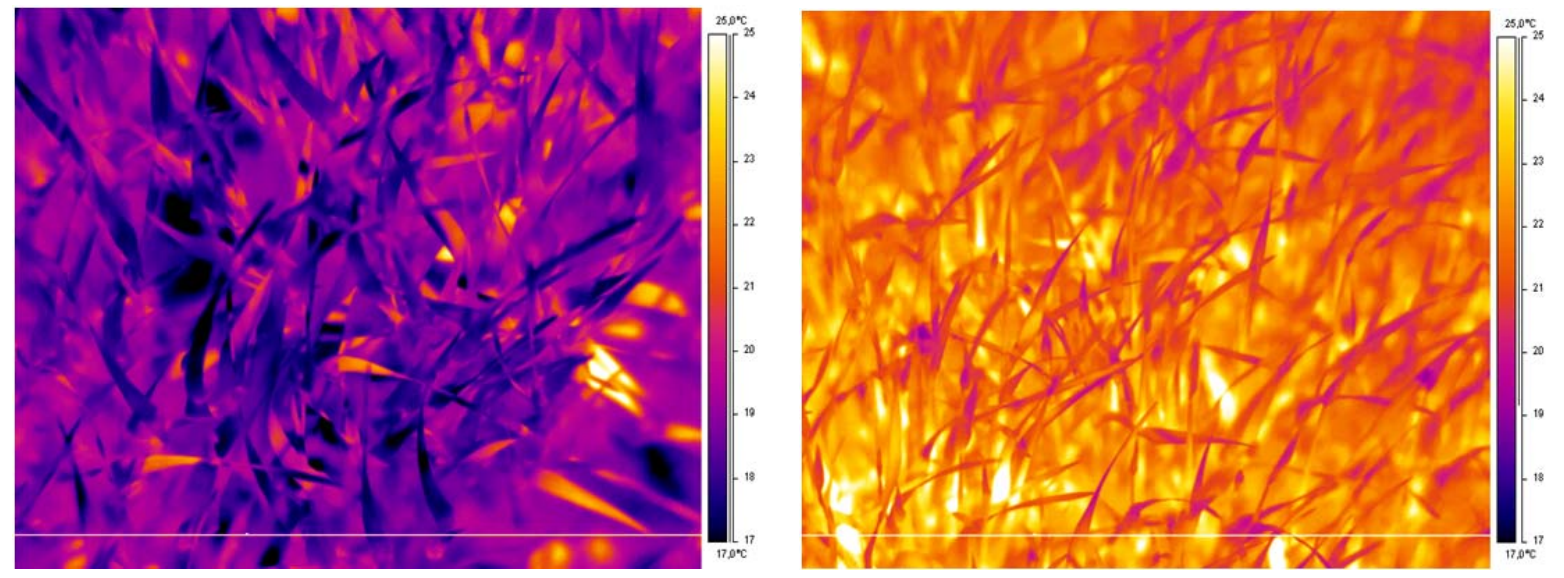

Figura 7. Fotografías térmicas del 30/04/2015. A la izquierda T100, a la derecha T70

Aparecieron diferencias significativas en el rendimiento final en todos los tratamientos salvo entre el T90 y T80. Como era de esperar, el mayor rendimiento se obtuvo en el tratamiento sin déficit. Entre el tratamiento T100 y el T70, la diferencia fue de $2210 \mathrm{~kg} / \mathrm{ha}$ con un ahorro de agua de $750 \mathrm{~m}^{3} / \mathrm{ha}$.

Tabla 5. Rendimientos simulados y reales de los tratamientos ensayados.

\begin{tabular}{|c|c|c|c|c|c|c|c|}
\hline \multirow{2}{*}{$\begin{array}{l}\text { TRATA - } \\
\text { MIENTO }\end{array}$} & \multirow{2}{*}{$\begin{array}{c}\text { Agua } \\
\text { neta de } \\
\text { riego } \\
\text { aplicada } \\
\left(\mathrm{m}^{3} / \mathrm{ha}\right)\end{array}$} & \multirow{2}{*}{$\begin{array}{l}\text { RENDIMIENTO } \\
\text { SIMULADO } \\
\text { MOPECO } \\
\text { (kg/ha) }\end{array}$} & \multicolumn{3}{|c|}{$\begin{array}{l}\text { RENDIMIENTO } \\
\text { REAL }\end{array}$} & \multirow{2}{*}{$\mathrm{Ys} / \mathrm{Yo}^{(3)}$} & \multirow{2}{*}{$\begin{array}{c}\text { EFICIENCIA } \\
\text { EN EL USO } \\
\text { DEL AGUA } \\
\text { DE RIEGO } \\
(\mathrm{kg} / \mathrm{mm})\end{array}$} \\
\hline & & & $\mathrm{kg} / \mathrm{ha}$ & $\mathrm{DV}^{(1)}$ & $C V^{(2)}$ & & \\
\hline No Deficit & 2856 & 8936 & 9440 & 698 & 7.4 & 0.95 & 33.05 \\
\hline T100 & 2506 & 8349 & 8614 & 458 & 5.3 & 0.97 & 34.37 \\
\hline T90 & 2251 & 7615 & 7620 & 362 & 4.8 & 1.00 & 33.85 \\
\hline T80 & 2002 & 7022 & 7362 & 169 & 2.3 & 0.95 & 36.77 \\
\hline T70 & 1753 & 6369 & 6404 & 492 & 7.7 & 0.99 & 36.53 \\
\hline
\end{tabular}

${ }^{(1)}$ SD: Desviación estándar; ${ }^{(2)} \mathrm{CV}$ : Coeficiente de variación; ${ }^{(3)}$ Ys/Yo: Rendimiento simulado/Rendimiento observado

En la Figura 8 se distingue que hay solape entre el T80 y T90, de ahí las no diferencias significativas entre ambos tratamientos (sus $\mathrm{ET}_{\mathrm{a}} / \mathrm{ET}_{\mathrm{m}}$ globales fueron 0.87 y 0.82 respectivamente). También se advierte que los rendimientos esperados al principio de la campaña y los simulados al final de la campaña han sido muy similares a los reales, salvo en el caso del T100, ya que al final sufrió estrés.

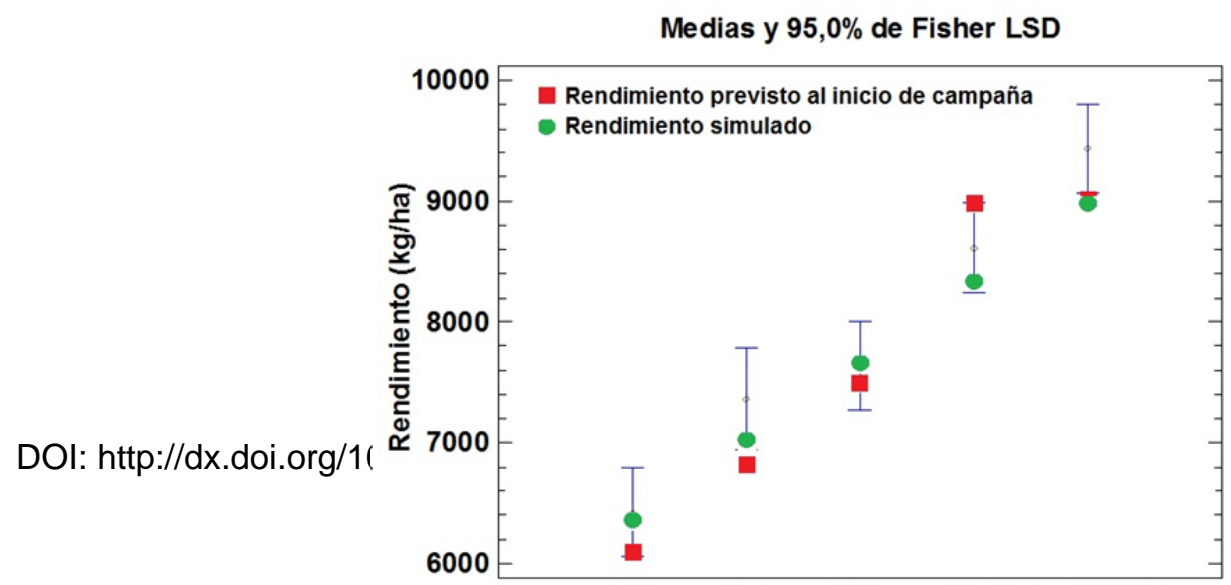


Figura 8. Rendimientos reales, simulados y previstos al inicio de la campaña.

Intermalta distingue 3 categorías en función del calibre del grano:

- Primeras: $>2.5 \mathrm{~mm}$

- Segundas: $2.2 \mathrm{~mm}$

- Terceras: $<2.2 \mathrm{~mm}$

Los granos catalogados como terceras directamente se derivan a pienso, no se utilizan para maltear, por tanto no interesan. Dentro de las "primeras" se distingue una subcategoría correspondiente a los granos que son mayores de $2.8 \mathrm{~mm}$. Ésta es la más relevante de todas.

Todos los tratamientos consiguieron un alto porcentaje de "primeras" (>95.5\%) habiendo diferencias significativas solo entre el T70 y el resto de tratamientos.

Dentro de la subcategoría $>2.8 \mathrm{~mm}$. aparecieron más diferencias significativas. Los mejores resultados los obtuvieron los tratamientos T90 y T80 con un 86 y $87 \%$ del total, seguidos del tratamiento sin déficit con un $83 \%$, el T100 con un $77 \%$ y el T70 con un $72 \%$. El mal resultado del T100 puede ser debido a que como siempre buscaba $E T_{a} / E T_{m}=1$, en la última etapa no quedaba agua y se castigó mucho el cultivo.

En "segundas", el orden ha sido el mismo, los mejores resultados los T90 y T80 (1.3\%) y el peor el T70 (2.5\%).

Todos los tratamientos tuvieron menos de un $1 \%$ de "terceras".

Todos los tratamientos consiguieron un contenido en proteína adecuado al marcado por la maltería. En concreto, todos los tratamientos salvo el sin déficit estuvieron dentro del rango óptimo (entre 11.5 y 12\%). Que el tratamiento sin déficit haya conseguido un porcentaje de proteína menor al $11.5 \%$ se justifica porque la producción real ha sido mayor a la esperada, y por tanto, el abonado se ha podido quedar un poco corto (el abonado fue distinto por cada tratamiento, se realizó en función al rendimiento esperado). No ha habido diferencias significativas entre los cuatro tratamientos deficitarios, sólo entre estos y el tratamiento sin déficit.

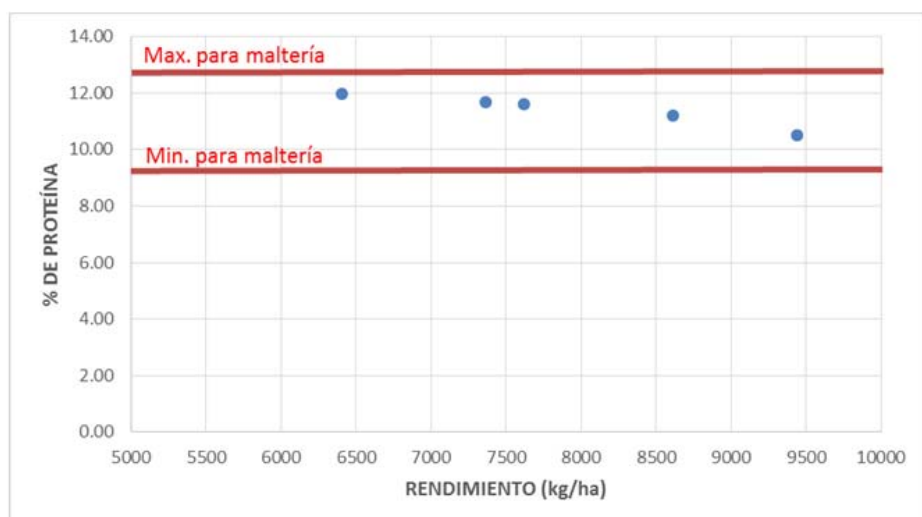

Figura 10. Porcentaje de proteína por rendimiento y tratamiento

\section{4- Conclusiones y recomendaciones}


La combinación de las metodologías ha permitido distribuir un volumen de agua limitado para cada tratamiento sin alcanzar un estrés severo global en ninguna de las etapas de desarrollo, logrando garantizar un alto rendimiento sin decremento de la calidad, pese a desconocer la evolución climática de la campaña de riegos.

Debido a la distribución de las lluvias y los parámetros climáticos reales, en algunas etapas, el déficit real ha sido distinto del objetivo previsto en un principio.

Los rendimientos reales han sido similares a los simulados por el modelo para cada tratamiento y a los esperados al inicio de la campaña. Se han encontrado diferencias significativas entre todos los tratamientos salvo entre el T90 y T80.

El porcentaje de proteína ha estado en el rango óptimo establecido por Intermalta.

\section{5- Bibliografía}

Abrha, B., Delbecques, N., Raes, D., Tsegay, A., Todorovic, M., Heng, L., Vanutrecht, E., Geerts, S., García-Vila, M., Deckers, S. (2012). Sowing strategies for barley (Hordeum vulgare L.) based on modelled yield response to water with AquaCrop. Expl. Agric., doi:10.1017/S0014479711001190.

Allen, R.G., Pereira, L.S., Raes, D., Smith, M. (1998). Crop Evapotranspiration: Guide- lines for Computing Crop Water Requirements. Irrigation and Drainage Paper No. 56. FAO, Italy.

Araya, A., Habtu, S., Hadgu, K.M., Kebede, A. Dejene, T. (2010). Test of AquaCrop model in simulating biomass and yield of water deficient and irrigated barley (Hordeum vulgare). Agricultural Water Management. 97 1838-1846.

Bleiholder, H., Weber, E., Lancashire, P.D., Feller, C., Buhr, L., Hess, M., Wicke, H., Hack, H., Meier, U., Klose, R., van den Boom, T., Stauss, R. (2001). Growth stages of mono-and dicotyledonous plants $\mathrm{BBCH}$ Monograph. 2nd edition. Ed. Meier, U. Federal Biological Research Centre for Agriculture and Forestry. Braunschweig, Germany.

Domínguez, A., Tarjuelo, J.M., de Juan, J.A., López-Mata, E., Breidy, J., Karam, F. (2011). Deficit irrigation under water stress and salinity conditions: The MOPECO-salt model. Agri Water Manage 98:1451-1461

Domínguez, A., de Juan, J.A., Tarjuelo, J.M., Martínez R.S., Martínez-Romero A. (2012). Determination of optimal regulated deficit irrigation strategies for maize in a semi-arid environment. Agric. Water Manage. 110, 67-77.

Domínguez, A., Martínez-Romero, A., Leite, K.N., Tarjuelo, J.M., de Juan, J.A., López-Urrea, R. (2013). Combination of typical meteorological year with regulated deficit irrigation to improve the profitability of garlic in central Spain. Agric.Water Manage. 130, 154-167.

Doorenbos, J., Kassam, A.H. (1979). Yield response to water. Irrigation and Drainage Paper No. 33. FAO, Italy.

Farahani, H.J., Izzi, G., Oweis, T.Y. (2009). Parameterization and evaluation of the AquaCrop model for full and deficit irrigated cotton. Agron. J. 101 (3), 469-476.

Heng L.K., Hsiao, T., Evett, S., Howell, T., Steduto, P. (2009). Validating the FAO AquaCrop model for irrigated and water deficient field maize. Agron. J., 101, 3, 488-498.

Juskiw, P.E., Jame, Y.W. \& Kryzanowski, L. (2001). Phenological development of spring barley in a short-season growing area. Agronomy Journal, 93, 370-379.

Kirby, E., Appleyard, M., \& Fellowes, M. (1982). Effect of sowing date on temperature response of leaf emergence and leaf size in barley, Plant Cell \& Environment, 5, 477-484.

López-Bellido L. (1991). Cultivos Herbáceos Extensivos. Cereales. Ediciones Mundi-Prensa. Madrid, España.

MAGRAMA (2016). Anuario de 2014. http://www.magrama.gob.es/estadistica/pags/anuario/2014/AE_2014_Completo.pdf Ministerio de Agricultura, Alimentación y Medio Ambiente. Consulta 5 abril 2016 
Moreno, M.A., Ortega, J.F., Córcoles, J.I., Martínez, A., Tarjuelo, J.M. (2010). Energy analysis of irrigation delivery systems: monitoring and evaluation of proposed measures for improving energy efficiency. Irrig. Sci. 28, 445-460.

Qureshi, Z.A., Neibling, H. (2009). Response of two-row malting spring barley to water cutoff under sprinkler irrigation. Agricultural Water Management. 96 141-148.

Sevacherian, V., Stern, V.M., Mueller, A.J. (1977). Heat accumulation for timing Lygus control pressures in a safflower-cotton complex. J. Econ. Entomol. 70, 399-402.

Stewart, J.I., Hagan, R.M., Pruitt, W.O., Kanks, R.J., Riley, J.P., Danilson, R.E., Franklin, W.T., Jackson, E.B. (1977). Optimizing crop production through control of water and salinity levels. Utah Water Res. Lab. PWRG 151-1. Utah, USA. 